\title{
Mathematical Model of Location Signal Reflections from The Substrate Surface of The Sea Observed By On-Board Radar
}

\author{
Gysberth Wattimena ${ }^{1}$, Vadim Nenashev ${ }^{2}$, Dmitry Shepeta ${ }^{3}$ \\ \{mauritswattimena@yahoo.com,nenashev.va@gmail.com, alex@shepeta.com\} \\ Saint-Petersburg State University of Aerospace Instrumentation, Saint-Petersburg, Russian Federation, \\ Rusia
}

\begin{abstract}
When testing onboard equipment of aircraft using for local monitoring, there is a need for mathematical models of signals reflected from the sea surface. The mathematical model of sea surface echoes based on the log-normal law is proposed, taking into account the spatiotemporal correlation function of the sea surface echo signal observed in the distance gate of the airborne locator of the aircraft. The proposed analytical expressions used to approximate the correlation functions are based on experimental data and a multidimensional logarithmic-normal model of fluctuations in the envelope of the echo signal. The model can be used in the design of onboard location complexes, in the interests of radar mapping, monitoring of environmental pollution, as well as for the synthesis of simulated sea-surface echo signals.
\end{abstract}

Keywords: Mathematical model, sea surface, correlation function, location signal, radar monitoring.

\section{Introduction}

Mathematical models of the sea surface echo are needed as in the synthesis, and in analyzing the operation of airborne radar aircraft carrying the radar monitoring.

When analyzing the performance of complex technical systems, practically the only method which allows you to explore the significant simplifications performance systems and to evaluate their effectiveness is the method of mathematical modeling of systems on a computer. During the computer simulation modeling algorithms, not only the system is needed, but also the algorithms for modeling the input processes of the studied system, which has a synthesis that is often no less complicated than the synthesis of the system itself. Therefore, the issues of synthesizing mathematical models that are adequate to the real input signals of on-board locators is extremely important, on the basis of which it is possible to synthesize effective algorithms for the simulation of systems on a computer.

The tasks of radar monitoring include assessment of the characteristics of the surrounding marine environment, determining the size, nature and extent of water surface contamination, determining the area of leakage of combustible and liquid chemicals in disaster areas; identification of zones of flooding and shallowing of the coasts of the seas and lakes; production of cartographic works on the sea surface, etc.

A successful solution to this list of monitoring tasks is determined by the capabilities of information radio electronic (primarily radar) systems capable of extracting information about the objects of observation contained in the characteristics of the echo signal. 


\section{The Mathematical Model Of The Sea Surface Echo Signal At The Input Of The Receiving Path Of The Aircraft}

The locating signal reflected from an extended object, in particular from the sea surface, is a random process. With a pulsed location mode with a $T_{R L S}$ period, this signal, observed at the distance gate of a receiver with duration of $\square_{g}$, is a segment of a random narrow-band process of duration $\square_{g}$, following the repetition period of the $T_{R L S}$.

When constructing a mathematical model for a narrowband process, one can construct either model of two quadrature $(U(t), V(t))$, or the envelope $A(t)$ and the phase $\Phi(t)$ of the echo signal. Since the data on the envelope of the echo signals of the sea surface are presented in the scientific literature devoted to experimental studies, we consider the process $(A(t), \Phi(t))$, replacing the continuous realization of the process by its discrete analogue $(A(t), \Phi(t))=\{A(t=(i-1) \square T), \Phi(t=(i-1) \square T)\} i=1,2, \ldots$, where $\square T$ is the discretization interval.

To determine the mathematical model, it is necessary to specify a multidimensional joint density (function) of the distribution of random variables $\left(\boldsymbol{A}_{N}, \boldsymbol{\Phi}_{N}\right)$ for an arbitrary set of indices, where $\boldsymbol{A}_{N}, \boldsymbol{\Phi}_{N}$ are $N$-dimensional vectors. In scientific sources, there is no information on the functional form of the distribution density $w\left(\boldsymbol{A}_{N}, \boldsymbol{\Phi}_{N}\right)$, but there are data on onedimensional laws of the envelope distribution of the echo signal $w(A)$ and on the correlation spectral characteristics of the envelope. As for the statistical characteristics of the phase vector, in experimental work, they are practically not represented. Therefore, the choice of the form $w\left(\boldsymbol{A}_{N}, \boldsymbol{\Phi}_{N}\right)$ is based only on the information regarding the type $w(A)$ and the correlation spectral characteristics of the echo signal envelope.

Various distribution densities were used as $w(A)$, the most common approximations of $w(A)$ in the form of Rayleigh distribution, Rayleigh-Rice distribution, Chi-square, $K$ distribution, log-normal distribution and some others. The log-normal distribution was most widely used, which not only agrees well with numerous experimental data but also allows the synthesis of effective algorithms for modeling the fluctuations of the signal envelope. Therefore, as the density distribution of the envelope of the sea surface echo, a log-normal distribution is received.

\section{The Density Of The Envelope Distribution Of A Localized Signal Reflected From The Sea Surface}

Consider the double indexing of the envelope samples, the sample $A_{i j}$ is the $j$-th count of the envelope in the $i$-th strobe of the range, $i=1,2, \ldots ; j=1,2, \ldots M$, that is, the count $A_{i j}$ is due to the reflection of the $i$-th location pulse of duration $\square_{3}$ from the $j$-th track, $M=E[\square g / \square T]$ is the number of envelope counts in the receiver's gate taken through sampling interval $\square T, E[\cdot]$ Antje function.

Such double indexing is convenient both in the consideration of the physics of the process and in the synthesis of modeling algorithms, that is, in the construction of the simulation model. When considering the correlation-spectral characteristics of the signal envelope, it is sufficient to use the two-dimensional marginal distribution density, which we write in the form

$$
\begin{gathered}
w\left(A_{i j}, A_{n m}\right)=\frac{1}{2 \pi \sigma_{i j} \sigma_{n m} A_{i j} A_{n m} \sqrt{1-r_{i j n m}^{2}}} \cdot \exp \left\{-\frac{1}{2\left(1-r_{i j n m}^{2}\right)} .\right. \\
\left.\left[\frac{1}{\sigma_{i j}^{2}} \ln ^{2} \frac{A_{i j}}{\bar{A}_{i j}}+\frac{1}{\sigma_{n m}^{2}} \ln ^{2} \frac{A_{n m}}{\bar{A}_{n m}}-2 r_{i j n m} \frac{1}{\sigma_{i j}} \ln \frac{A_{i j}}{\bar{A}_{i j}} \cdot \frac{1}{\sigma_{n m}} \ln \frac{A_{n m}}{\bar{A}_{n m}}\right]\right\},
\end{gathered}
$$


Where $\bar{A}_{i j}$ and $\sigma_{i j}$ are the distribution parameters associated with the mathematical expectation $\widetilde{A}_{i j}$ and variance $\widetilde{\sigma}_{i j}^{2}=\widetilde{D}_{i j}$ of the distribution by the relations.

$$
\left\{\begin{array}{l}
\bar{A}_{i j}=\tilde{A}_{i j} / \sqrt{1+\left(\tilde{\sigma}_{i j} / \tilde{A}_{i j}\right)^{2}}=\widetilde{P}_{i j}\left(1+K_{i j}^{2}\right), \\
\sigma_{i j}^{2}=\ln \left(1+\left(\tilde{\sigma}_{i j} / \widetilde{A}_{i j}\right)^{2}\right)=\ln \left(1+K_{i j}^{2}\right) .
\end{array}\right.
$$

Where $K_{i j}=\tilde{\sigma}_{i j} / \tilde{A}_{i j}$ is the coefficient in amplitude of variation of the log-normal distribution, and $\widetilde{P}_{i j}$ is the average power of the reflections, determined from the known radar formulas.

$$
\tilde{P}_{i j}=\frac{P_{i} \cdot \lambda_{i}^{2} \cdot c \cdot \tau_{3} \cdot \eta_{i} \cdot G_{i j}^{2} \cdot \Delta \varphi}{2 \cdot(4 \pi)^{3} \cdot L_{i j}^{3} \cdot \cos \theta_{i j}} \cdot 10^{0,1 \sigma_{i j}^{0}},
$$

where $P_{i}$ is the transmitter power at the $i$-th pulse emission, the reflection of which causes the echo signal in the $j$-th position of the $i$-th received packet, $\square_{3}$ - duration probe pulse, $c$ - the light velocity, $\mathrm{L}_{i j}$ - the slant range to the surface element ( $i, j$-th element), which causes the echo signal, $\square_{i j}$ - the angle of sight in the vertical plane of $i, j$-th element of surface, $\eta_{i}$ - loss factor, $\sigma_{i j}^{0}$ specific EPR of $i, j$-th element of surface $G_{i j}$ - the antenna gain in the direction of $i$, $j$-th element, $\Delta \varphi$ i - beamwidth of antenna at the half power level. In the expression ${ }^{(3)}{ }_{S_{i j}} \approx\left(c \cdot \tau_{3} \cdot L_{i j} \cdot \Delta \varphi \cdot 10^{0,1 \sigma_{i j}^{\circ}}\right) /\left(2 \cdot \cos \theta_{i j}\right)$ - EPR of $i, j$-th element of a surface in $\mathrm{m}^{2}$.

The parameter $r_{i j n m}$ in the expression (1) is equal to

$$
r_{i j m m}=\frac{\ln \left(1+K_{i j} K_{n m} R_{i j m m}\right)}{\sqrt{\ln \left(1+K_{i j}^{2}\right) \ln \left(1+K_{n m}^{2}\right)}},
$$

where $R_{i j n m}$ is the correlation coefficient between the $j$-th count of the envelope of the $i$-th strobe and the $m$-sample of the envelope of the $n$-th strobe, $i, n=1,2, \ldots ; j, m=1,2, \ldots M$. The physical meaning of the parameter $r_{i j n m}$ is the correlation coefficient between the logarithm of the $j$-th sample of the envelope of the echo signal at the $i$-th sounding and the logarithm of the $m$-th envelope count at $n$-th sounding. Accordingly, for $i=n$ and $j=m$, the correlation coefficients $R_{i j n m}=R_{i j i j}=R_{n m n m}=1$ and $r_{i j n m}=r_{i j i j}=r_{n m n m}=1$.

Scientific sources on experimental studies of sea-surface echoes, as a rule, contain information not about the space-time characteristics of the signal, but only information about the cross sections of the space-time correlation function. The information is given on the spatial correlation function $R^{(I I)}(\Delta l)$ and on the time correlation function $R^{(B)}(\tau)$, where $\Delta l$ is the horizontal distance between two sections (lanes of the range) of the sea surface, and $\tau$ is the time interval between the echo- signals from the same sea surface area. 
In the far field of observation, the correlation between the $j$-th and $m$-th counts of the echo within the gate $\square_{g}, j, m=1,2, \ldots M$, is mainly determined only by the spatial diversity of the sea surface regions that cause these echoes. Therefore, we can assume that $R_{i j i m}=R^{(I)}(\Delta l)$ is a spatial correlation function, i.e.

$$
\begin{aligned}
R_{i j i m} & =R^{(I)}\left(\Delta l=|j-m| \Delta T c /\left(2 \cos \bar{\theta}_{i}\right)\right)= \\
& =R^{(I)}\left(\tau^{\prime}=|j-m| \Delta T\right)=R^{(I)}\left(\tau^{\prime}\right),
\end{aligned}
$$

where $R^{(I)}\left(\tau^{\prime}\right)$ is the spatially normalized correlation function or a function of the correlation coefficients of the inter-period fluctuations (signal fluctuations within the gate $\square_{g}$ ), $\tau^{\prime}=|j-m| \Delta T$ - the distance between samples in the gate of the receiving device, $\mathrm{c}$ - speed of light. In expression (5) it is assumed that in the far field of observation of the sea surface the viewing angle $\theta_{i j} \approx \bar{\theta}_{i}$ for $i=1,2, \ldots ; j=1,2, \ldots M$.

When the antenna is stationary or when tracking the observed sea surface area, the correlation function $R_{i j n j}=R_{j}^{(B)}(\tau)=R^{(B)}(\tau)$ is a time correlation function

$$
R_{i j n j}=R^{(B)}\left(\tau=|i-n| T_{R L S}\right)=R^{(B)}(\tau)
$$

There exists a correlation function of inter-period fluctuations. In the far field of observation within the gate $\square_{g}$, the distance between the echo signals $\tau^{\prime}=|j-m|$ $\triangle T \leq \square \square g<<T_{R L S}$, therefore the time correlation function within the gate is practically equal to ${ }^{\text {unity }}{ }_{i j i m}=R_{i}^{(B)}\left(\tau^{\prime}=|j-m| \Delta T\right) \approx R_{i}^{(B)}\left(\tau_{g}\right) \approx R^{(B)}\left(\tau_{g}\right) \approx 1$, for $i=1,2, \ldots ; j=1,2, \ldots M$.

Thus, under the conditions indicated above, the relations are as follows:

$$
\left\{\begin{array}{l}
R_{i j n j}=R_{i m n m}=R_{i n}^{(B)}=R^{(B)}\left(|i-n| T_{\mathrm{PЛС}}\right)=R^{(B)}(\tau), \\
R_{i j i m}=R_{n j n m}=R_{j m}^{(\Pi)}=R^{(\Pi)}(|j-m| \Delta T)=R^{(I)}\left(\tau^{\prime}\right),
\end{array}\right.
$$

When the relations (7) are satisfied, the equalities for counting the logarithm of the envelopes will also be fulfilled with great accuracy, existing for the parameters $r_{i j n m}$,

$$
\left\{\begin{array}{l}
r_{i j n j}=r_{i m n m}=r_{i n}^{(B)}=r^{(B)}\left(|i-n| T_{\mathrm{PЛC}}\right)=r^{(B)}(\tau), \\
r_{i j i m}=r_{n j n m}=r_{j m}^{(\Pi)}=r^{(\Pi)}(|j-m| \Delta T)=r^{(\Pi)}\left(\tau^{\prime}\right) .
\end{array}\right.
$$

Performing the equations (8) considerably simplifies the synthesis of simulation algorithms fluctuations echo sea surface. We assume that the equalities (8) are exact without imposing any conditions, then equalities (7) will also be satisfied with great accuracy in the far zone, for which, as indicated above, $\theta_{i j} \approx \bar{\theta}_{i}$ and $R^{(B)}\left(\square_{g}\right) \approx 1, i=1,2, \ldots ; j=1,2, \ldots M$.

It is impossible to reconstruct the spatiotemporal correlation function of the echo signals of the sea surface $R_{i j n m}$ from its cross-sections. However, it can be defined in such a way that its cross sections coincide with the cross-sections of the real space-time correlation function and, in addition, it will be related to the space-time correlation function of the logarithm of the envelope by the relation (4). We define $R_{i j n m}$ in the form 


$$
\begin{aligned}
& R_{i j n m}=\frac{1}{K_{i j} K_{n m}} \cdot\left(\exp \left(r_{i j n m} \cdot \sqrt{\ln \left(1+K_{i j}^{2}\right) \cdot \ln \left(1+K_{n m}^{2}\right)}\right)-1\right) \\
& =\frac{1}{K_{i j} K_{n m}} \cdot\left(\exp \left(r_{j m}^{(I)} r_{i n}^{(B)} \cdot \sqrt{\ln \left(1+K_{i j}^{2}\right) \cdot \ln \left(1+K_{n m}^{2}\right)}\right)-1\right) .
\end{aligned}
$$

Such an approximation allows one to reproduce spatially temporal sections of the spacetime correlation function accurately (without methodological error) and, in addition, allows the method of simulation of echo signals of the sea surface to be used for the method of multidimensional nonlinear shaping filters, which dramatically increases the speed of the modeling process. In this case, the statistical characteristics of the simulation model coincide with the known experimental characteristics of the sea surface echoes.

\section{Specific Epr And The Variation Coefficient Of The Sea Surface}

It is necessary to set the parameters in the expression (1) within the practical application of a mathematical model, which in turn are determined by the characteristics of the on-board locator, the observational conditions of the sea surface, and its overall state.

The characteristics of the locator and the conditions of observation are necessary to be specified by the system designer. The parameter $\bar{A}_{i j}$ is determined by, which in turn is calculated through $\widetilde{A}_{i j}$, which in turn $\widetilde{P}_{i j}$ is determined by the expression (2). Therefore, to calculate $\widetilde{P}_{i j}$, it is necessary to specify the specific EPR of the sea $\sigma_{i j}^{o}$. Currently, there is no general theory regarding the reflections of location signals from the sea surface, which would give a general expression for $\sigma^{0}$. Therefore, empirical formulas are used in the design of the location systems to determine $\sigma^{0}$.

In the scientific sources the following empirical dependence of $\sigma^{0}$ is proposed for small angles of view of $\square_{i j}$ and relatively calm sea.

$\sigma_{i j}^{o}=10\left[1,6 \lg \left(\theta_{i j}+0,5\right)+\frac{0,9}{\lambda_{i}}\left(W_{i}-\frac{\psi_{i}}{\pi}-\sin \psi_{i}\right)-5\right]$.

where $\square_{i j}$ - in degrees, $\psi_{i}$ - in radians, $\square_{i}$ - in inches, $W_{i}$ - in points, $\sigma^{0}$ - in decibels per square meter. It is argued that the formula (10) for $0 \leq \square_{i j} \leq 45^{\circ}, 0 \leq \psi_{i} \leq \pi$, $0,8 \mathrm{inch} \leq \lambda \leq 10 \mathrm{inch}, 0 \leq W \leq 6$ points gives an accuracy in the range $\pm 3 \mathrm{~dB} / \mathrm{m}^{2}$. Formula (10) is valid for vertical polarization, and for the horizontal, the calculated values $\sigma^{0}$ must be reduced by $7 \ldots 15 \mathrm{~dB} / \mathrm{m}^{2}$.

The variation coefficient $K_{i j}$ depends on $\theta_{i j}, \psi_{i}, W_{i}, L_{i j}$ and $\tau_{3}$. The dependence of the variation coefficient to the duration of the probing signal $\tau_{3}$ are mild, and the slant range $L_{i j}$ is observed only at small distances of less than 1 to $6 \mathrm{~km}$. In the scientific sources, the following empirical formula for $K_{i j}$ is suggested.

$$
\begin{gathered}
K_{i j}=K\left(\theta_{i j}, \psi_{i}, W_{i}\right)= \\
=0,52-\left[0,17+\left(W_{i}-5\right) \cdot 10^{-2}\right]\left(1-\frac{\psi_{i}}{\pi}\right) \sin \left(9 \theta_{i j}\right),
\end{gathered}
$$

where $\square_{i j}$ - in degrees, $\psi_{i}$ - in radians, $W_{i}$ - in points. Expression (11) is valid for $0 \leq \square_{i j} \leq 10^{\circ}, 0 \leq \psi_{i} \leq \pi, 0 \leq W \leq 6$ points. In the far zone, when $\square_{i j}$ is small, we can assume a 
constant $K_{i j}$, independent from $\square_{i j}, \psi_{i}$ and $W_{i}$, and equal to $K_{A}=\sqrt{(4-\pi) / \pi} \approx 0,52$ which is the coefficient of variation of the Rayleigh distribution.

\section{The Spatial Correlation Function Of The Radar Signal Reflected From The Sea Surface}

Let us first consider the spatial correlation function $R_{j m}^{(\Pi)}=R^{(\Pi)}(|j-m| \Delta T)=R^{(\Pi)}\left(\tau^{\prime}\right)$, where $\tau^{\prime}=|j-m| \Delta T$. In the far zone $\theta_{i j}=\bar{\theta}_{i}$ and $K_{i j}=K_{i}$, i.e. for any $i$ the coefficient of variation from the track number does not depend, therefore.

$$
\begin{aligned}
& R_{i j i m}=\frac{1}{K_{i j} K_{i m}}\left(\exp \left(r_{i j i m} \sqrt{\ln \left(1+K_{i j}^{2}\right) \ln \left(1+K_{i m}^{2}\right)}\right)-1\right)= \\
& =\frac{1}{K_{i}^{2}}\left(\exp \left(r_{j m}^{(I I)} \sqrt{\ln \left(1+K_{i}^{2}\right) \ln \left(1+K_{i}^{2}\right)}\right)-1\right)= \\
& =\frac{1}{K_{i}^{2}}\left(\left(1+K_{i}^{2}\right)^{r_{j m}^{(I I)}}-1\right)
\end{aligned}
$$

In scientific sources for the centimeter wave band, the dependence of the correlation coefficient of the echo signal in the distance gate $R^{(I)}\left(\tau^{\prime}=\tau_{3}\right)=R^{(I)}\left(\tau_{3}\right)$ is given between the envelope samples spaced by the duration of the probing pulse $\square_{3}$

$$
R^{(I)}\left(\tau_{3}\right)=1-\left(0,3-7 \cdot 10^{-3} \cdot \bar{\theta}_{i}+0,18 \sin \psi_{i}-\frac{0,02}{W_{i}}\right) \frac{\tau_{3}}{1,5},
$$

Where $\bar{\theta}_{i}$ and the angle of the waves $\square_{i}$ is in degrees, the sea wave $W_{i}$ is in points, $\square_{3}$ is in microseconds, the formula can be used with the following restrictions, $0.5^{\circ} \leq \bar{\theta}_{i} \leq 10^{0}$, $0^{\circ} \leq \square_{i} \leq 180^{\circ}, 1$ point $\leq W_{i} \leq 6$ points, 1 microsecond $\leq \square_{3} \leq 3$ microseconds. Since we can assume that $\square_{i}$ and $W_{i}$ do not change significantly during operation $\bar{\theta}_{i}$, the correlation coefficient $R^{(I)}\left(\tau_{3}\right)$ does not depend on the gate number for small values, so the index $i$ is omitted here and $R_{i}^{(\Pi)}\left(\tau_{3}\right)=R^{(\Pi)}\left(\tau_{3}\right)$.

We approximate the spatial correlation function of the curve $R_{j m}^{(\Pi)}=R^{(\Pi)}(|j-m| \Delta T)$ defined by expression (8),

$R_{j m}^{(\Pi)}=\frac{1}{K_{i j} K_{i m}}\left[\exp \left(\sqrt{\ln \left(1+K_{i j}^{2}\right) \ln \left(1+K_{i m}^{2}\right)} r_{j m}^{(\Pi)}\right)-1\right]$,

where $r_{j m}^{(n)}=r^{(n)}(|j-m| \Delta T)$ is the function corresponding to the correlation function of the normal Markov process. In the far zone $K_{i j}=K_{i m}=K_{i}$, we can even consider $K_{i j}=K_{i m}=K_{i}=K$, which is exactly true for a fixed antenna, therefore

$$
\begin{aligned}
& R_{j m}^{(I I)}=R^{(I)}(|j-m| \Delta T)= \\
& =\frac{1}{K_{i}^{2}}\left[\exp \left(r_{j m}^{(I)} \ln \left(1+K_{i}^{2}\right)\right)-1\right]= \\
& =\frac{1}{K_{i}^{2}}\left[\left(1+K_{i}^{2}\right)^{r_{j m}^{(I)}}-1\right]
\end{aligned}
$$


For a Markov process of the first order $r_{j m}^{(\Pi)}=r\left(\tau^{\prime}\right)$ has the form

$$
\begin{aligned}
\left.r_{j m}^{(I)}\right) & r^{(I)}(|j-m| \Delta T)= \\
& =r^{(I)}\left(\tau^{\prime}\right)= \\
=\exp ( & (-\alpha|j-m| \Delta T)= \\
= & \exp \left(-\alpha \tau^{\prime}\right),
\end{aligned}
$$

where $\square$ is a certain coefficient, which is determined from the above expressions by equating $\tau^{\prime}=\tau_{3}$

$$
\alpha=-\frac{1}{\tau_{3}} \ln \frac{\ln \left(1+K_{i}^{2} R^{(\Pi)}\left(\tau_{3}\right)\right)}{\ln \left(1+K_{i}^{2}\right)} .
$$

Calculations $\tau_{0,5}^{(\Pi)}$ and $R^{(\Pi)}\left(\square_{3}\right)$ show that in the far zone $\square$ can be regarded as a constant that depends weakly on the conditions of observation of the sea surface.

\section{The Time Correlation Function Of The Location Signal Reflected From The Sea Surface}

We now turn to the consideration of temporal correlation function $R_{i n}^{(B)}=R^{(B)}\left(|i-n| T_{R L S}\right)=R^{(B)}\left(\tau=|i-n| T_{R L S}\right)=R^{(B)}(\tau)$ which is the correlation functions of inter-period fluctuations of the envelope of the sea surface. We approximate $R_{i n}^{(B)}$ the curve corresponding to the expression (8)

$$
\begin{aligned}
& R_{i n}^{(B)}=R^{(B)}\left(|i-n| T_{\text {РЛС }}\right)=R^{(B)}(\tau)= \\
& =\frac{1}{K_{i} K_{n}}\left[\exp \left(\sqrt{\ln \left(1+K_{i}^{2}\right) \ln \left(1+K_{n}^{2}\right)} r^{(B)}(\tau)\right)-1\right],
\end{aligned}
$$

whereas $r^{(B)}(\square)$, which reflects the characteristic features of the curves, it is expedient to use a curve of the form.

$$
r^{(B)}(\tau)=C_{1} e^{-\alpha_{1}|\tau|} \cos (\gamma \tau)+C_{2} e^{-\alpha_{2}|\tau|}+C_{3} e^{-\alpha_{3}|\tau|}
$$

where $C_{1}, C_{2}, C_{3}, \square_{\square} \square \square_{\square} \square \square_{\square} \square \square$ are non-negative constants, with $C_{1}+C_{2}+C_{3}=1$.

For practice, the approximation of the expression (19) is sufficient, i.e. the fluctuations in the envelope of the sea surface are well described by a logarithmically normal Markov process of the fourth order.

\section{Conclucions}

Mathematical models of sea surface echoes, based on experimental data, make it possible to study the operation of airborne locators of aircraft under conditions that are as close as possible to the full-scale experiment. In the construction of such models, as the experimental 
data, the researcher has at his disposal, the data on the one-dimensional law of probability distribution and on the correlation spectral characteristics of the envelope fluctuations.

The log-normal distribution law is the most common law of distribution of the envelope of the echo signal used in practice. It not only fits well with experimental histograms of distributions but is also easy to write and convenient in theoretical calculations.

When approximating the correlation functions of the sea surface echoes, it is necessary to take into account the law of the envelope distribution. In particular, for a log-normal model, exponential and exponential-cosine curves should approximate the fluctuations of the envelope logarithm, and not the correlation functions themselves. Such an approximation, in particular, makes it possible to synthesize simulation models of envelope fluctuations free from methodological errors. In this case, the space-time correlation function of the signals is not factorized.

It follows from the models proposed in the work that the echo signals of the sea surface, corresponding to its sections, spaced at a distance far greater than the probe signal, are correlated, which agrees well with known experimental facts.

\section{References}

[1] Kulemin G.P. Radar interference from the sea and land radars in centimeter and millimeter ranges. Proceedings of the International Scientific and Technical Conference (Paper). Kiev: Academy of Sciences of Ukraine, NGO Kvant. Edition 1, 1994. pp. 23-29.

[2] Trunc G.V, Gejrge S.F. Detection of Targets in Non-Gaussion Sea Clutter.// IEEE Transactions on Aerospace and Electronic Systems, 1970, Vol AES-6, N. 5, pp. 620628

[3] Bessonov A.A, Sesin A.E, Shepeta A.P. Mathematical and simulation models of sea surface echoes. National Association of Aviation Devices. Aerospace instrument Russia. Series 2, Avionics. Issue 4. St. Petersburg. 2005. pp. 52-69.

[4] Sayama S, Sekine M. Log-normal, log-Weibull and K-distributed sea clutter, IEICE Trans. 2002. Commun, Vol. E85-B, pp. 1375-1381.

[5] Wattimena G. M. Mathematical models of the location signals reflected from the underlying surfaces of the earth and the sea. Modeling of X-ray images for ecosystem monitoring tasks. Modern research of social problems. 2017. Vol. 8. No. 4-2. from. pp. 49-58.

[6] Podoplekin Y. F., Nenashev V. A., Shepeta D. A. Modeling of Input Signals to Onboard Radar, due to the Accumulated Reflections of the Probing Signal from the Underlying Surfaces of the Earth and Sea. Morskoi vestnik [Bulletin of Marine], 2016, no. 4, pp. 69-71 (In Russian).

[7] Shepeta D.A, Sesin A.E. Mathematical model of echo signals of the sea surface, observed by aircraft airborne locators. Information-control systems. 2010. № 2. pp. 21-25.

[8] Shepeta A. P., Nenashev V. A. Modeling Algorithm for SAR Image Based on Fluctuations of Echo Signal of the Earth's Surface. Proc. of SPIE Remote Sensing, Toulouse, France, 2015, vol. 9642, pp. 96420X-1-9642OX-8.

[9] Keith D. Ward, Robert J.A. Tough, and Simon Watts. Sea Clutter: Scattering the KDistribution and Radar Performance, IET Radar, Sonar and Navigation Series 20. The Institute of Engineering and Technology: London. 2006, 452 p. 
[10] Shepeta D.A. Development of mathematical models and synthesis of algorithms for modeling input signals of onboard information processing and control systems. Thesis for the degree of Candidate of Technical Sciences / St. Petersburg, 2000.

[11] Junming Xia, Weihua Bai, Danyang Zhao. First shipborne GNSS-R campaign for receiving low elevation angle sea surface reflected signals. IEEE International Geoscience and Remote Sensing Symposium (IGARSS). 2016.

[12] Yongqiang Qi, Bo Zhang, Dongkai Yang. Three dimensional modeling of GNSS ocean reflection signal. IEEE International Geoscience and Remote Sensing Symposium (IGARSS). 2016.

[13] Min-Ho Ka, Aleksandr I. Baskakov, Alexey Artemenko. Effect of the Local Backscattering Pattern of the Sea Surface to the Reflected Signal of a Precision Airborne Radar Altimeter at Low Altitude. IEEE Geoscience and Remote Sensing Letters - Volume: 13, Issue: Sept. 2016.

[14] Roman Galas, Tilo Schone, Marija Cokrlic. On precise GNSS-based sea surface monitoring systems. ELMAR, 55th International Symposium. 2013.

[15] Blaunstein N. Sh., Sergeev M. B.. Shepeta A. P. Prikladnye aspekty elektrodinamiki [Applied Aspects of Electrodynamics]. Saint-Petersburg, Agraf +, 2016. 272 p. (In Russian).

[16] Isakov V. I., Podoplekin Y. F., Shepeta D. A. Markov Model of the Fluctuations of the Amplitudes and Durations of the Echo-signals of Large Surface Objects. Morskoi vestnik [Bulletin of Marine], 2016, no. 3 (59), pp. 49-50 (In Russian).

[17] Verba V. S. Spravochnik po radiolokatsii [Handbook of Radar]. M. I. Skolnick ed. In 2 books. Book 2. Moscow, Tekh-nosfera Publ., 2015. 680 p. (In Russian). 